\title{
Transactions
}

Cite this: Dalton Trans., 2011, 40, 3671

wWW.rsc.org/dalton

PAPER

\section{One electron oxidation of triferrocenylmethanol: synthesis, metal atom dynamics, electron delocalization, and the crystal structure of $\left[\mathrm{Fe}_{3} \mathrm{COH}\right]^{+}$ $\mathrm{PF}_{6}{ }^{-} \uparrow$}

\author{
Holger Butenschön, ${ }^{a}$ Jingxiang Ma, ${ }^{a}$ Constantin G. Daniliuc, ${ }^{b}$ Israel Nowik ${ }^{c}$ and Rolfe H. Herber*c \\ Received 16th September 2010, Accepted 4th January 2011 \\ DOI: $10.1039 / \mathrm{codt01248c}$
}

\begin{abstract}
The title compound 2 was prepared and its crystal structure was determined at $100 \mathrm{~K}$. The neat solid was examined by temperature dependent ${ }^{57} \mathrm{Fe}$ Mössbauer effect (ME) spectroscopy over the interval 92 $<T<318 \mathrm{~K}$, and evidences two diamagnetic $\mathrm{Fe}$ (II) sites and one paramagnetic $\mathrm{Fe}(\mathrm{III})$ site. The latter shows spin-lattice relaxation, but there is no evidence of electron delocalization among the three iron sites in the above temperature interval. The mean-square-amplitude-of-vibration of the diamagnetic iron site has been determined from the recoil-free fraction ME resonance, and compared to the neutral $\mathrm{Fc}_{3} \mathrm{COH}$ homologue (1). The ME dynamical data are in good agreement with the $U_{\mathrm{i}, \mathrm{j}}$ value at $100 \mathrm{~K}$ extracted from the crystallographic results. The ME parameters at $5 \mathrm{~K}$ have also been determined with the sample compound embedded in a paraffin wax matrix as well as pelletized with $\mathrm{BN}$.
\end{abstract}

\section{Introduction}

Ferrocene is considered to be an aromatic compound as much of its chemistry, in particular electrophilic aromatic substitution, very much resembles that of an activated benzene. ${ }^{1,2}$ However, significant differences between these compounds are found in the fact that ferrocene is a three dimensional molecule with all the associated stereochemical implications, and that ferrocene, in contrast to benzene, is easily oxidized with formation of the ferrocenium cation. ${ }^{1,3}$ These similarities and differences have caused interest in the triferrocenylmethyl system, ${ }^{4-11}$ which is the ferrocene analogue to Gomberg's triphenylmethyl system. ${ }^{12,13}$

One important question in this context is that of an interaction between the ferrocenyl substituents in triferrocenylmethane derivatives. In a recent study from these laboratories the metal atom dynamics of a series of structurally related triferrocenylmethyl complexes were reported in detail. ${ }^{14}$ As a consequence of these studies we became interested in the question of whether it would be possible to subject triferrocenylmethanol (1) to a one electron oxidation, and in how far the resulting positive charge of the cation of the oxidation product $\mathbf{2}$ would be localized on one of the iron atoms or if there was some type of charge

Institut für Organische Chemie, Leibniz Universität Hannover, D30167, Hannover, Germany. E-mail: holger.butenschoen@mbox.oci.unihannover.de; Fax: +49 511 762-4616; Tel: +49511 762-4661

${ }^{b}$ Institut für Anorganische und Analytische Chemie, Technische Universität Braunschweig, Hagenring 30, D-38106, Braunschweig, Germany. E-mail: c.daniliuc@tu-braunschweig.de;Tel:+49531391-5382

'Racah Institute of Physics, The Hebrew University of Jerusalem, 91904, Jerusalem,Israel. E-mail: herber@vms.huji.ac.il; Fax: +972 2 6586347; Tel: +97226584244

$\dagger$ CCDC reference number 804181. For crystallographic data in CIF or other electronic format see DOI: $10.1039 / \mathrm{c} 0 \mathrm{dt} 01248 \mathrm{c}$ delocalization to the other two iron atoms. It has now been possible to isolate the related hexafluorophosphate complex $\mathbf{2}$ of the corresponding $\left[\mathrm{Fc}_{3} \mathrm{COH}\right]^{+}$cation and to examine the crystal structure, electron delocalization properties and metal atom dynamics of this complex.

\section{Experimental}

\section{General. ${ }^{15}$ - Compound 2}

At $-35^{\circ} \mathrm{C} \mathrm{AgPF}_{6}(25.2 \mathrm{mg}, 0.1 \mathrm{mmol})$ in dichloromethane $(2 \mathrm{~mL})$ was added to triferrocenylmethano $l^{8}(58.4 \mathrm{mg}, 0.1 \mathrm{mmol})$ in dichloromethane $(10 \mathrm{~mL})$. The color of the solution changed from light red to dark green. The mixture was stirred for $10 \mathrm{~min}$ at $25{ }^{\circ} \mathrm{C}$. Precipitated silver was removed by filtration through Celite ${ }^{\circledR}$, which was washed with dichloromethane $(3 \times 10 \mathrm{~mL})$. After the removal of solvent at reduced pressure, the product was obtained as a dark green solid (71 mg, $0.1 \mathrm{mmol}, 97 \%)$. Recrystallization from dichloromethane at $25{ }^{\circ} \mathrm{C}$ gave a dark green crystal (m. p. $178.2-179.2{ }^{\circ} \mathrm{C}$ ). IR (ATR): $\tilde{v}=3108$ (w), 3088 (w), 2927 (w), 2364 (w), 2324 (w), 2269 (w), 2234 (w), 2175 (w), $2163(\mathrm{w}), 1996(\mathrm{w}), 1740(\mathrm{w}), 1558(\mathrm{w}), 1423(\mathrm{w}), 1391(\mathrm{w})$, $1343(\mathrm{w}), 1303(\mathrm{w}), 1262(\mathrm{w}), 1212(\mathrm{w}), 1106(\mathrm{~m}), 1060(\mathrm{w})$, 1035 (w), 1003 (m), 838 (s), 730 (s) $\mathrm{cm}^{-1} \cdot{ }^{1} \mathrm{H}$ NMR $(400 \mathrm{MHz}$, acetone- $\left.d_{6}\right): \delta=4.70(\mathrm{~s}, 10 \mathrm{H}, \mathrm{CpH}), 5.63(\mathrm{~s}, 1 \mathrm{H}, \mathrm{OH}), 5.69$ $5.72\left(\mathrm{AA}^{\prime} \mathrm{BB}^{\prime}, 8 \mathrm{H}, \mathrm{HOCCp} H\right), 17-18$ (broad, unresolved signal, ca. $10 \mathrm{H}, \mathrm{CpHFe}^{+} \mathrm{CpR}$; signal disappears upon standing for $6 \mathrm{~d}$ ) ppm. ${ }^{13} \mathrm{C}$ NMR (100 MHz, acetone- $\left.d_{6}\right): \delta=75.4(\mathrm{FeCp}), 79.5$ (br, $\mathrm{COH}$ ), 81.3 (br, $\mathrm{HOCCCHCH}), 89.5$ (HOCC) ppm. MS (70 eV): $m / z(\%)=584(20)\left[\mathrm{Fc}_{3} \mathrm{COH}^{+}\right], 583(49), 582(100), 398(27)$ $\left[\mathrm{Fc}_{3} \mathrm{COH}^{+}-\mathrm{FcH}\right], 302$ (11), 287 (27), $236(30), 84$ (11), 73 (28), 71 (12), 69 (14), 57 (47), 55 (19). 


\section{Crystal structure analysis}

A single crystal was obtained from dichloromethane at $25{ }^{\circ} \mathrm{C}$. $\mathrm{C}_{32} \mathrm{H}_{30} \mathrm{Cl}_{2} \mathrm{~F}_{6} \mathrm{Fe}_{3} \mathrm{OP} ; M_{r}=813.98 \mathrm{~g} \mathrm{~mol}^{-1}$; crystal system monoclinic; space group $P c ; a=10.7296(2), b=25.7495(4), c=11.2096(2)$ $\AA ; \alpha=90, \beta=97.289(2), \gamma=90^{\circ} ; V=3071.98(9) \AA^{3} ; Z=4 ; \rho=$ $1.760 \mathrm{~g} \mathrm{~cm}^{-3} ; \mu=13.874 \mathrm{~mm}^{-1}$; crystal size $0.12 \times 0.10 \times 0.90 \mathrm{~mm}^{3}$; $F(000)=1644 ; T=100(2) \mathrm{K} ; \mathrm{Cu}-\mathrm{K} \alpha$ radiation $(\lambda=1.54184 \AA): \theta$ range 3.43 to $76.00^{\circ}$; reflections collected/unique $43728 / 10257$ $(R($ int $)=0.0537)$; completeness of data $\theta=72.50^{\circ}(99.9 \%)$; index ranges $-13 \leq h \leq 11,-32 \leq k \leq 32,-13 \leq l \leq 14$; absolute structure parameter 0.007(3); empirical absorption correction (multi-scan), no extinction correction, direct methods, full-matrix least-squares refinement on $F^{2}$, goodness-of-fit on $F^{2}=1.107, R_{1}=0.0434(I>$ $\left.2 \sigma_{I}\right), \mathrm{w} R_{2}=0.1114, R$-indices [all data] $R_{1}=0.0469, \mathrm{w} R_{2}=0.1131$, final difference electron density 0.888 and $-0.778 \mathrm{e}^{-3}$.

Data were recorded on a Oxford Diffraction Xcalibur diffractometer at low temperature using mirror-focussed $\mathrm{Cu}-\mathrm{K} \alpha$ radiation. The structure was refined anisotropically using the program SHELXL-97. ${ }^{16}$ Hydrogen atoms were included using a riding model. The hydrogen atoms from $\mathrm{OH}$ groups were refined freely but using distance restraints (DFIX).

Special features: the Flack parameter for the noncentrosymmetric structure 2 was refined to $0.007(3)$. The dichloromethane molecule is disordered over two positions. The atoms of the minor component were refined isotropically.

\section{Temperature-dependent ${ }^{57} \mathrm{Fe}$ Mössbauer effect spectroscopy (ME)}

This technique was employed as previously described. ${ }^{14,17}$ Two independently synthesized samples ( $a$ and $b$ ) were examined in this study. In order to ensure that no sample loss was incurred during the lengthy data acquisition times, the transmission counting rate was monitored before and after each temperature-dependent data point. Temperature was monitored using the Daswin software. ${ }^{18}$ Spectrometer calibration was effected using a $20 \mathrm{mg} \mathrm{cm}^{-2} \alpha-\mathrm{Fe}$ absorber at room temperature, and all isomer shifts are reported with respect to the centroid of such calibration spectra.

\section{Results and discussion}

One electron oxidation triferrocenylmethanol (1) with silver hexafluorophosphate afforded $\mathbf{2}$ in $97 \%$ yield as dark green, air stable crystals.

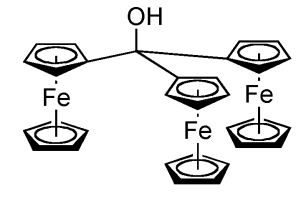

1

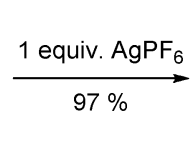

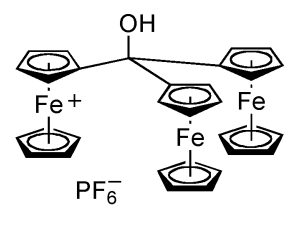

2
Recrystallization from dichloromethane gave crystals suitable for an X-ray structure analysis (Fig. 1). The compound 2 crystallized in the monoclinic space group $\mathrm{P} c$ with two molecules in the asymmetric unit. One molecule of dichloromethane per molecule of $\mathbf{2}$ disordered over two positions was found in the lattice.

The bond lengths between the iron atoms and the respective cyclopentadienyl carbon atoms clearly show that the ferrocenyl

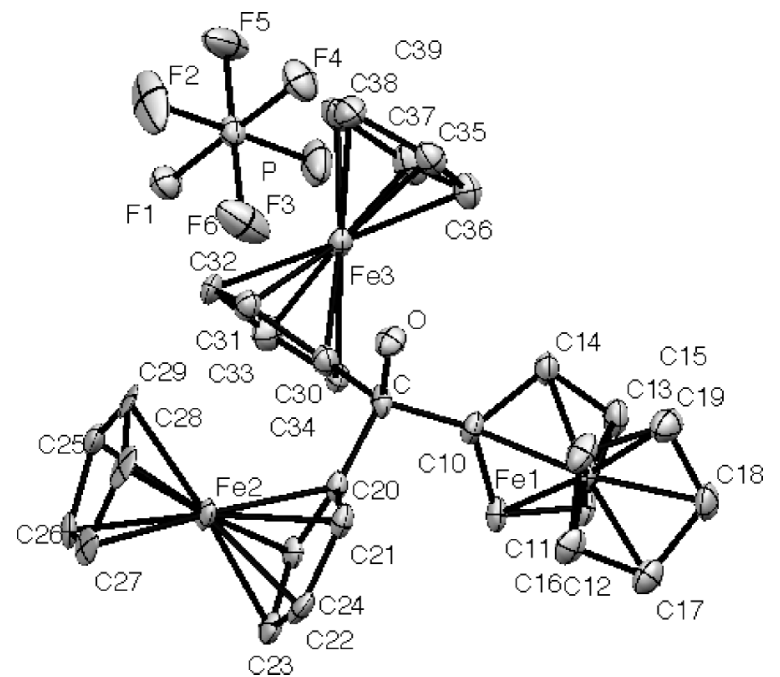

Fig. 1 Structure of 2 in the crystal. ${ }^{19}$ Diagram of one of the two independent molecules $\mathbf{2}$ with thermal displacement parameters drawn at $50 \%$ probability. The hydrogen atoms and the disordered dichloromethane molecule were omitted for clarity. Selected bond lengths [pm] and angles $\left[^{\circ}\right]$ : $\mathrm{C}-\mathrm{O}$ 142.7(5), $\mathrm{C}-\mathrm{C} 10$ 151.8(5), C-C20 151.7(6), C-C30 151.1(6), Fe1-C10 205.1(4), Fe1-C11 205.8(5), Fe1-C12 205.4(5), Fe1-C13 205.1(5), Fe1-C14 204.0(5), Fe1-C15 205.2(5), Fe1-C16 206.2(5), Fe1-C17 205.8(5), Fe1-C18 204.7(5), Fe1-C19 205.9(5), $\mathrm{Fe} 2-\mathrm{C} 20$ 213.6(4), Fe2-C21 210.7(4), Fe2-C22 207.9(4), Fe2-C23 208.6(5), Fe2-C24, 210.6(4), Fe2-C25 210.2(4), Fe2-C26 208.4(5), Fe2-C27 209.8(5), Fe2-C28 211.1(5), Fe2-C29 211.1(5), Fe3-C30 205.9(5), Fe3-C31 203.7(5), Fe3-C32 204.2(5), Fe3-C33 205.3(5), Fe3-C34 205.1(5), Fe3-C35 206.2(5), Fe3-C36 205.7(5), Fe3-C37 205.6(5), Fe3-C38 205.0(5), Fe3-C39 204.3(5); O-C-C10 110.9(4), O-C-C20 107.7(4), O-C-C30 112.2(4).

units containing $\mathrm{Fe} 1$ and $\mathrm{Fe} 3$ are different from those with $\mathrm{Fe} 2$. While the average distance between the iron atom and the cyclopentadienyl carbon atoms is $205.3 \mathrm{pm}$ for $\mathrm{Fel}$ and $205.1 \mathrm{pm}$ for $\mathrm{Fe} 3$, it is $210.2 \mathrm{pm}$ for $\mathrm{Fe} 2$. This observation is in accord with 2 consisting of two $\mathrm{Fe}$ (II) and one $\mathrm{Fe}$ (III) sites with the cyclopentadienyl ligands being less strongly bound to $\mathrm{Fe}(\mathrm{III})$. Remarkably, the bond lengths between $\mathrm{Fe} 2$ and the cyclopentadienyl carbon atoms are significantly longer than those in ferrocenium hexafluorophosphate, which shows an average value of $205.1 \mathrm{pm}$, the mean standard deviations of the analysis being significantly higher than in the case of $2 .{ }^{20}$ Although there is no obvious explanation for this large difference, we note that the cyclopentadienyl ligands in ferrocenium hexafluorophosphate adopt an almost perfect staggered conformation (twist angle $33.2^{\circ}$ ), while the corresponding value for the ferrocenium substituent in $\mathbf{2}$ is $-22.9^{\circ}$, indicating a deviation from the staggered conformation of $\mathrm{ca} .10^{\circ}$.

The electrochemical properties of $\mathbf{2}$ were investigated by cyclic voltammetry $(\mathrm{CV})$ and compared with those of unoxidized 1. As expected, the cyclic voltammograms are very similar, differences are attributed to the fact that $\mathbf{2}$ is much less soluble in acetonitrile as compared to $\mathbf{1}$. The difference in concentration results in different currents for the two compounds, both plots are given on the same scale. Both of the cyclic voltammograms show quasi-reversible oxidation-reduction waves, which are in accord with the redox processes of the ferrocene moieties (Fig. 2), the respective data are listed in Table 1. The fact that three redox processes were observed 
Table 1 Cyclic voltammetry data of $\mathbf{1}$ and $\mathbf{2}$ (potentials in $\mathrm{V} v s . \mathrm{Fc} / \mathrm{Fc}^{+}$, $v=100 \mathrm{mV} \mathrm{s}^{-1}, T=25^{\circ} \mathrm{C}, c(\mathbf{1})=2 \mathrm{mmol} \mathrm{L}^{-1}, c(\mathbf{2})=0.2 \mathrm{mmol} \mathrm{L}^{-1}, 0.1 \mathrm{~mol}$ $\mathrm{L}^{-1} \mathrm{Bu}_{4} \mathrm{NPF}_{6}$ in acetonitrile)

\begin{tabular}{lrrrr}
\hline Compd. & $E_{\mathrm{pa}}[\mathrm{V}]$ & $E_{\mathrm{pc}}[\mathrm{V}]$ & $\Delta E[\mathrm{~V}]$ & $E_{1 / 2}[\mathrm{~V}]$ \\
\hline $\mathbf{1}$ & 0.307 & 0.217 & 0.090 & 0.262 \\
& 0.146 & 0.065 & 0.081 & 0.106 \\
$\mathbf{2}$ & -0.026 & -0.112 & 0.086 & -0.069 \\
& 0.327 & 0.240 & 0.087 & 0.284 \\
& 0.165 & 0.081 & 0.084 & 0.123 \\
& -0.015 & -0.099 & 0.084 & -0.059 \\
\hline
\end{tabular}
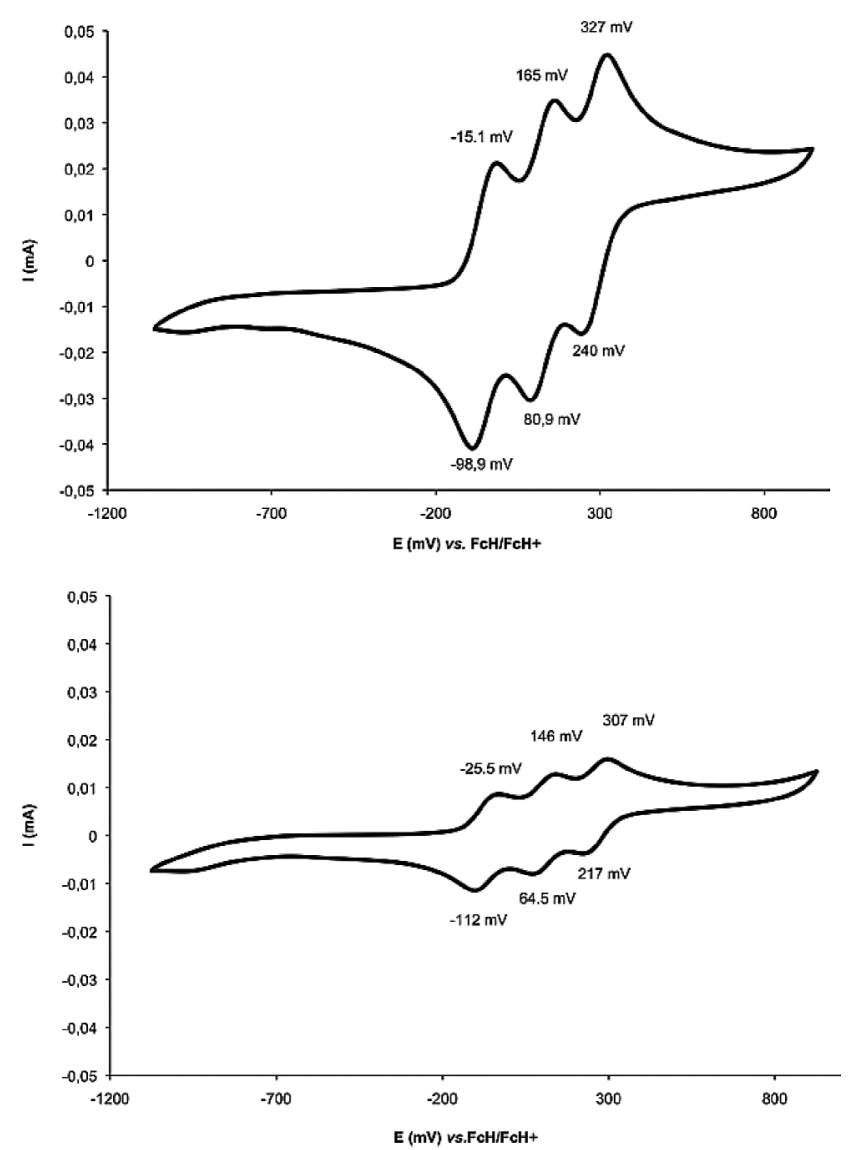

Fig. 2 Cyclic voltammograms of $\mathbf{1}$ (top) and $\mathbf{2}$ (bottom). Potential vs. $\mathrm{FcH} / \mathrm{FcH}^{+}$. For further details see Table 1 .

for $\mathbf{1}$ and $\mathbf{2}$ indicates that the oxidation products contain four different ferrocene/ferrocenium moieties, presumably 1, 2, and products of the two further possible oxidation steps. For complexes $\mathbf{1}$ and 2, two of the oxidation reactions take place at positive potentials relative to ferrocene/ferrocenium, and all the $\Delta E$ values are almost identical; however, 2 has relatively higher $E_{1 / 2}$, than $\mathbf{1}$.

The ME spectra of $\mathbf{1}$ as received consist of a three-line pattern of which the two outside lines are identified with the un-oxidized (diamagnetic) iron atoms, and the central broad resonance is typical of the oxidized (paramagnetic) metal atoms of the structure.

A typical spectrum, that of sample 2(a), is shown in Fig. 3. This identification immediately leads to two significant conclusions: (i) the ratio of the areas of the diamagnetic to paramagnetic sites is not exactly $2: 1$ as might have been expected, but rather is of the order of 2.85 and slightly temperature dependent. The most likely

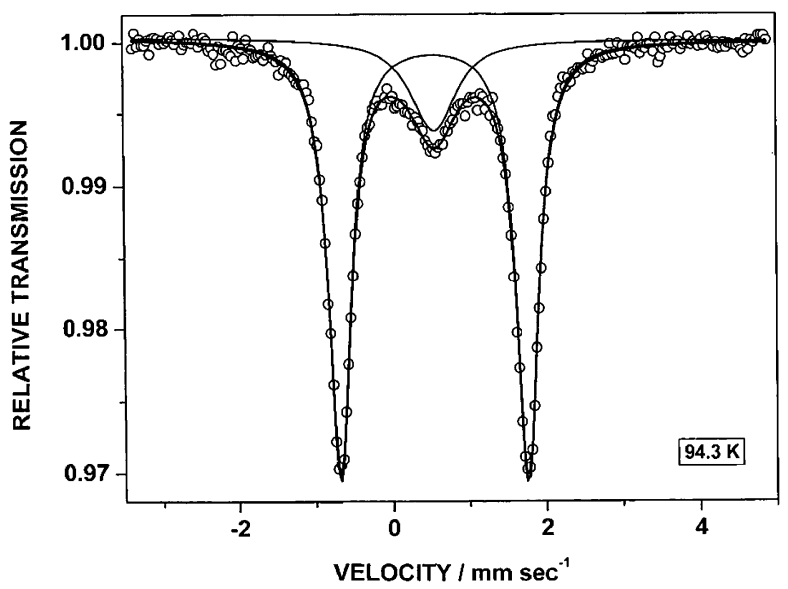

Fig. $3{ }^{57} \mathrm{Fe}$ Mössbauer spectrum of $\mathrm{Fc}_{3} \mathrm{COH}^{+} \mathrm{PF}_{6}{ }^{-}$(2) at $94.3 \mathrm{~K}$. The isomer shift scale is with respect to an $\alpha$-Fe absorber spectrum at room temperature.

explanation of this observation is not that the recoil-free fraction of the diamagnetic site is larger than that of the paramagnetic site, as will be further detailed below, but that the as-examined sample includes a small impurity of the neutral tris compound 1 resulting from the synthetic procedure. (ii) The line widths (FWHM) of the diamagnetic resonance $\left(0.30-0.36 \mathrm{~mm} \mathrm{~s}^{-1}\right)$ are only modestly different from the values observed for the neutral triferrocenylmethanol (1) reported earlier $\left(0.23-0.26 \mathrm{~mm} \mathrm{~s}^{-1}\right){ }^{14}$ This small broadening may be due to the small difference in the hyperfine parameters of $\mathbf{1}$ and those observed for the non-oxidized Fe atoms in 2. From this observation it can be unambiguously concluded that there is no electron delocalization between the diamagnetic and paramagnetic iron sites in this complex in the temperature range $90<T<300 \mathrm{~K}$. This interpretation is also consistent with the agreement of the hyperfine parameters (isomer shift, IS, and quadrupole splitting, QS) associated with the diamagnetic sites in $\mathbf{2}$ and the neutral complex $\mathbf{1}$ reported earlier. $^{14}$

As noted previously, ${ }^{21}$ the paramagnetic resonance line is significantly broader than the diamagnetic resonance, and the FWHM of this line decreases with increasing temperature. This behavior is characteristic of spin relaxation, ${ }^{22}$ and these line widths have been analyzed in terms of a relaxation mechanism, as discussed previously. Since the spin state of the paramagnetic site has not been determined independently, the computer data fitting has assumed an arbitrary value for $H_{\text {int }}$ so that the absolute values of the relaxation rate (RLX) are not significant in the present context. However, the temperature-dependence of this (arbitrary) rate permits the evaluation of the power law describing this relaxation process, and the relevant results over the range $92<T<300 \mathrm{~K}$ are summarized in Fig. 4. The data at higher temperatures are uncertain due to the small effect magnitudes associated with these higher temperatures (vide infra). The 2.6 power of the temperature dependence suggests that the spin relaxation process is not simply of the spin-lattice type ${ }^{23}$ but may, in fact, obtain due to several simultaneous relaxation processes.

In this context, it is appropriate to note that an earlier examination of $\mathrm{Fc}_{3} \mathrm{C}^{+} \mathrm{BF}_{4}{ }^{-}$(compound 4 of ref. 14) shows only the Mössbauer resonance lines characteristic of diamagnetic $\mathrm{Fe}(\mathrm{II})$; that is, in this compound there is no significant electron withdrawal 


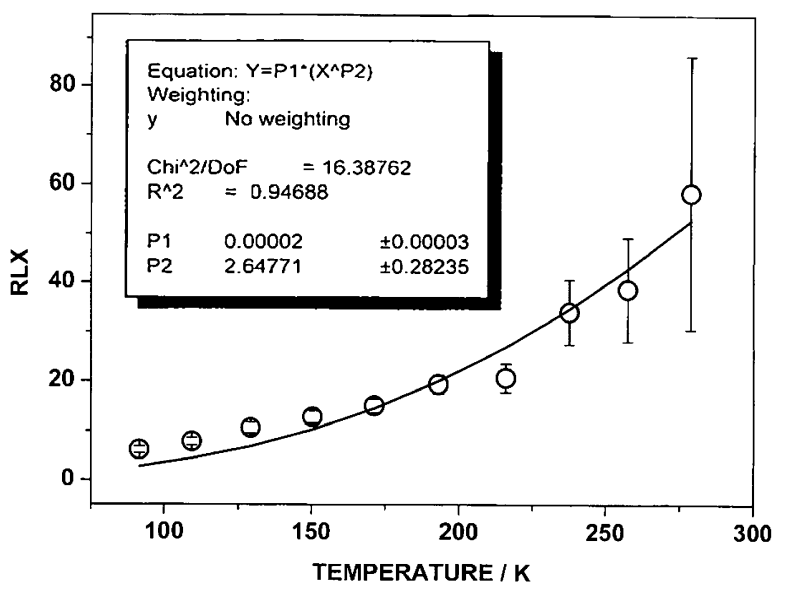

Fig. 4 Temperature dependence of the relaxation rate of $\mathbf{2}$ (paramagnetic site). The ordinate (RLX) is the arbitrary relaxation rate as discussed in the text.

from any of the three iron sites, in contrast to the behavior of $\mathbf{2}$ detailed above.

The hyperfine parameters extracted from the above data are summarized in Table 2, which also includes the temperature dependence of the $I S$ parameter and the recoil-free fraction. With respect to the former, the $I S$ of the two sites at $90 \mathrm{~K}\left(I S_{\mathrm{D}}\right.$ and $\left.I S_{\mathrm{P}}\right)$ are essentially identical, in consonance with the earlier observation ${ }^{24}$ confirming the fact that removal of an electron from the metal atom in these systems, where the major bonding interaction of the metal atom involves the $\pi$-electrons of the ring system, has negligible effect on the electron density at the metal atom nuclear position. The $M_{\text {eff }}$ value $e^{25}$ for the metal atoms is on the order of $88 \pm 16$ daltons reflecting the covalency of the metal atom ligation. The relatively large error arises from the insensitivity of $I S$ on $T$. The $Q S$ parameter of the diamagnetic site is nearly temperature independent in the range $90<T<260 \mathrm{~K}$.

Turning next to the dynamical aspects of the metal atom in 2 it should be noted that the Mössbauer data fitting program calculates the relative area under the resonance curve for the diamagnetic and the paramagnetic iron sites. From this information one can calculate the temperature-dependence of the recoilfree fraction for each site, $f$, as a function of temperature over the interval $90<T<302 \mathrm{~K}$. This temperature dependence, summarized in Table $3,-\operatorname{dln}\left[A_{\mathrm{D}}(T) / A(90)\right]$, is $(8.92 \pm 0.07) \cdot 10^{-3}$ $\mathrm{K}^{-1}$, cc: 0.995 for 11 data points for the diamagnetic site. For the paramagnetic site, the temperature interval was taken only over the range $171<T<302 \mathrm{~K}$ due to the large errors associated with the low temperature data. For this interval, $-\operatorname{d} \ln \left[A_{\mathrm{P}}(T) / A(90)\right]$ is $(9.94 \pm 0.18) \times 10^{-3} \mathrm{~K}^{-1}$ cc: 0.991 for 7 data points, which is
Table 3 Derived parameters for sample 2(a) neat and compound $\mathbf{1}^{14}$ Abbreviations see Table 2

\begin{tabular}{llll}
\hline Sample & a neat & a $10 \mathrm{t} \mathrm{BN}$ & Compd. $\mathbf{1}^{14}$ \\
\hline$-\mathrm{d} I S_{\mathrm{D}} / \mathrm{d} T\left[\mathrm{~mm} \mathrm{~s}^{-1} \mathrm{~K}^{-1} \times 10^{4}\right]$ & $3.62(6)$ & $4.96(8)$ & $3.32(2)$ \\
$-\mathrm{d} \ln A_{\mathrm{D}} / \mathrm{d} T\left[\mathrm{~K}^{-1} \times 10^{3}\right]$ & $8.92(7)$ & $7.75(36)$ & $5.86(4)$ \\
$-\mathrm{d} I S_{\mathrm{P}} / \mathrm{d} T\left[\mathrm{~mm} \mathrm{~s}^{-1} \mathrm{~K}^{-1} \times 10^{4}\right]$ & $5.46(34)$ & $5.38(25)$ & - \\
$-\mathrm{d} \ln A_{\mathrm{P}} / \mathrm{d} T\left[\mathrm{~K}^{-1} \times 10^{3}\right]$ & $9.94(19)$ & $6.42(5)$ & - \\
$k^{2}<x_{\text {ave }}^{2}>_{M}, 100 \mathrm{~K}$ & 1.05 & - & $M, 173 \mathrm{~K}: 1.00(1)$ \\
$k^{2}<x_{\text {ave }}{ }^{2}>_{X}, 100 \mathrm{~K}$ & 0.99 & - & $X, 173 \mathrm{~K}: 1.04(6)$ \\
\hline
\end{tabular}

$\sim 10 \%$ larger than that for the diamagnetic site, suggesting that the paramagnetic site is slightly less strongly ligated to the $\mathrm{Cp}$ moieties than the latter. This is in accord with the observation of a longer distance of the $\mathrm{Fe}(\mathrm{III})-\mathrm{Cp}$ bond as compared to the $\mathrm{Fe}(\mathrm{II})-\mathrm{Cp}$ bond.

The temperature dependence of $f$ also permits the calculation ${ }^{26}$ of the parameter $F=k^{2}\left\langle x_{\text {ave }}^{2}\right\rangle$, where $k$ is the wave vector of the $14.4 \mathrm{keV}$ gamma ray and $\left\langle x_{\text {ave }}{ }^{2}\right\rangle$ is the square of the metal atom vibrational amplitude. This latter parameter can also be extracted from the crystallographic $U_{\mathrm{i}, \mathrm{j}}$ parameter. To compare the two results it is necessary to make the assumption ${ }^{27,28}$ that the ME $\ln f$ value extrapolates to $\sim 0$ as $T \rightarrow 0 \mathrm{~K}$. The comparison of the $F$ values extracted from the ME data and the value extracted from the single crystal X-ray data at $100 \mathrm{~K}$ is summarized graphically in Fig. 5, from which it is seen that agreement is quite satisfactory, given the experimental uncertainties involved; that is $F_{\mathrm{X}, 100}=1.05$, while $F_{\mathrm{M}, 100}=0.99$. The practical consequence of this agreement is that it is now possible to calculate the root-mean-square (rms) amplitude of vibration at all temperatures in the noted interval.

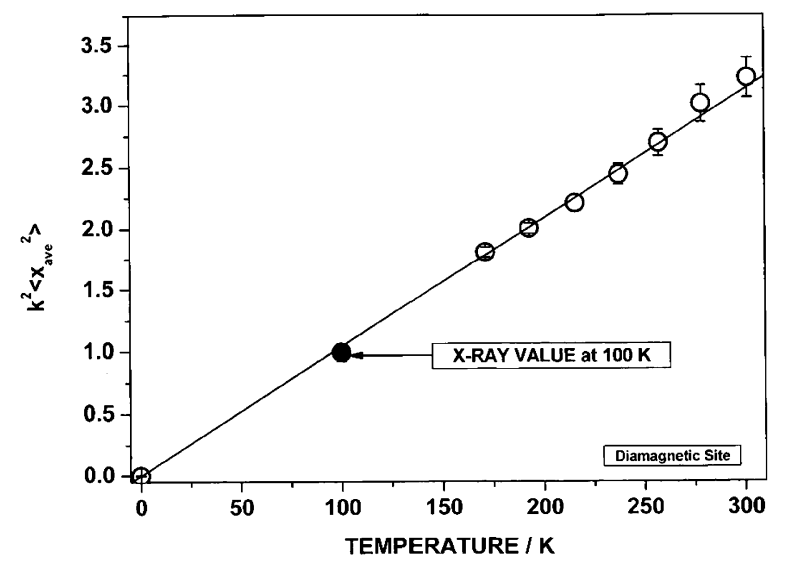

Fig. 5 Comparison of the ME $k^{2}<x_{\text {ave }}^{2}>$ values for the diamagnetic site in 2 and that extracted from the single crystal X-ray data at $100 \mathrm{~K}$ (filled point).

Table $2{ }^{57} \mathrm{Fe}$ Mössbauer hyperfine parameters of two independent samples of compound 2. $D=$ diamagnetic Site; $P=$ paramagnetic Site; T = tons pressure on sample (released); $\mathrm{BN}$ = boron nitride pellet; wax = paraffin wax pellet; compd $\mathbf{1}$, see ref. 1

\begin{tabular}{|c|c|c|c|c|c|c|c|c|c|c|}
\hline Sample & $\mathrm{a}$ & $\mathrm{a}$ & $\mathrm{a}$ & $\mathrm{a}$ & $\mathrm{b}$ & $\mathrm{b}$ & $\mathrm{b}$ & $\mathrm{b}$ & $\mathrm{b}$ & Compd 1 \\
\hline Param & neat & $10 \mathrm{~T} \mathrm{BN}$ & $10 \mathrm{~T} \mathrm{BN}$ & epoxy & neat & wax & wax & wax & $5 \mathrm{~T} \mathrm{BN}$ & neat \\
\hline$T / \mathrm{K}$ & 91.5 & 5 & 90 & 93 & 94.3 & 5 & 90 & 100 & 93.5 & 90 \\
\hline$I S_{\mathrm{D}}\left[\mathrm{mm} \mathrm{s}^{-1}\right]$ & $0.545(3)$ & 0.529 & $0.519(2)$ & $0.536(4)$ & 0.537 & $0.527(2)$ & $0.534(8)$ & $0.518(4)$ & $0.527(3)$ & $0.524(2)$ \\
\hline$Q S_{\mathrm{D}}\left[\mathrm{mm} \mathrm{s}^{-1}\right]$ & $2.428(3)$ & 2.414 & $2.411(2)$ & $2.392(4)$ & 2.433 & $2.408(2)$ & $2.388(8)$ & $2.426(4)$ & $2.464(3)$ & $2.398(2)$ \\
\hline$I S_{\mathrm{P}}\left[\mathrm{mm} \mathrm{s}^{-1}\right]$ & $0.544(3)$ & $0.545(5)$ & $0.524(7)$ & $0.477(22)$ & $0.537(17)$ & $0.537(4)$ & $0.536(28)$ & $0.524(23)$ & $0.518(14)$ & \\
\hline
\end{tabular}


Table 4 Root-mean-square-amplitude-of vibration of the iron atom for the diamagnetic site in $\mathbf{2}(\mathrm{a})$ and $\mathbf{1}$ in $\mathrm{pm}$

\begin{tabular}{lll}
\hline$T / \mathrm{K}$ & Compd. 1 & Compd. 2(a) \\
\hline 100 & 10.6 & 14.3 \\
150 & 12.4 & 17.4 \\
200 & 14.6 & 20.1 \\
250 & 16.5 & 22.5 \\
300 & 18.5 & 24.6 \\
\hline
\end{tabular}

This is done for 2(a) and $\mathbf{1}$ at four temperatures in Table 4. It is clear from these data that the ligation of the metal atom in the diamagnetic site of $\mathbf{2}$ is significantly weaker than it is in the neutral compound 1.

As noted above, in 2a the paramagnetic iron site appears to have a slightly smaller Mössbauer lattice temperature, ${ }^{25} \Theta_{\mathrm{M}}$, as determined from the temperature dependence of the IS parameter, than the diamagnetic site. The single crystal X-ray data shows that there are two distinct $\mathrm{Fe}-\mathrm{C}_{\mathrm{Cp}}$ distances observed; two at 205.1 and $205.9 \mathrm{pm}$ and one at $213.6 \mathrm{pm}$, and thus the single paramagnetic site is presumably less strongly ligated than the diamagnetic site, in consonance with the $M E \ln A$ data cited above.

It was deemed appropriate to examine the paramagnetic compound at about $5 \mathrm{~K}$ in a low temperature (closed cycle) cryostat. To effect this measurement, it was necessary to immobilize the sample in a vertical position, and to accomplish this it was attempted to embed the sample powder in an epoxy resin. However, this procedure resulted - completely unexpectedly - in the observation that the ratio of the diamagnetic to paramagnetic resonance was increased from its previous value $(2.8$ at $93 \mathrm{~K}$ ) to roughly four times this value. Normally, the epoxy component of commercial resin, which involves an epichlorohydrin group as well as triethylene tetramine in the hardener, is considered an oxidizing agent, which would have reduced the diamagnetic to paramagnetic ratio, rather than what is observed experimentally. However, it has been noted, ${ }^{29,30}$ in the presence of traces of water the oxidation of the epoxide to the corresponding carboxylic acid has been shown to occur. It is thus inferred that when neat $\mathbf{2}$ is mixed with commercial epoxy resin and allowed to harden, some of the paramagnetic iron sites are reduced to a diamagnetic state.

To solve the problem of supporting neat 2 in a form which could be examined in our low temperature cryostat (which requires vertical orientation of the sample), the neat powder (sample b) was mixed with paraffin wax, warmed just above the wax melting point, and then allowed to cool to room temperature. The resulting pellet was examined both at 5 and $90 \mathrm{~K}$ in this cryostat, and the results are reported in Table 2.

These $I S_{\mathrm{D}}$ and $Q S_{\mathrm{D}}$ parameters are essentially identical to those noted for sample (a) at $91.5 \mathrm{~K}$ and confirm the fact that the wax immobilization preserves the identity of the pristine sample. Moreover, the diamagnetic iron hyperfine parameters in (b) are essentially identical to those observed for the neutral triferrocenylmethanol (1). ${ }^{14}$

In this context, it was also attempted to achieve a pellet configuration of $\mathbf{2}$ by mixing the neat powder with $\mathrm{BN}$ and

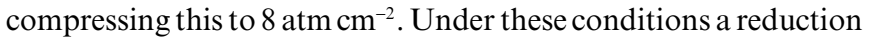
of about $16 \%$ of the diamagnetic to paramagnetic ratio at $\sim 90 \mathrm{~K}$ was observed suggesting the possibility of a minor crystallographic rearrangement on the imposition of pressure on the neat sample.
Finally, it is instructive to compare the dynamical properties of the two kinds of iron atoms in neat 2(a) and in the BN pellet compressed to 10 tons. These data are summarized in Table 3 and shown graphically in Fig. 6 and Fig. 7. In the neat sample, the temperature-dependence of the diamagnetic site $(D)$ is slightly smaller than that observed for the paramagnetic $(P)$ site, whereas in the pelletized sample the reverse appears to obtain. Although the two sets of temperature dependencies are very similar, it can be again concluded that exposing neat 2 to a pressure of 10 tons (about $8 \mathrm{~atm} \mathrm{~cm}^{-2}$ ) causes a crystallographic phase change even when the applied pressure is relieved.

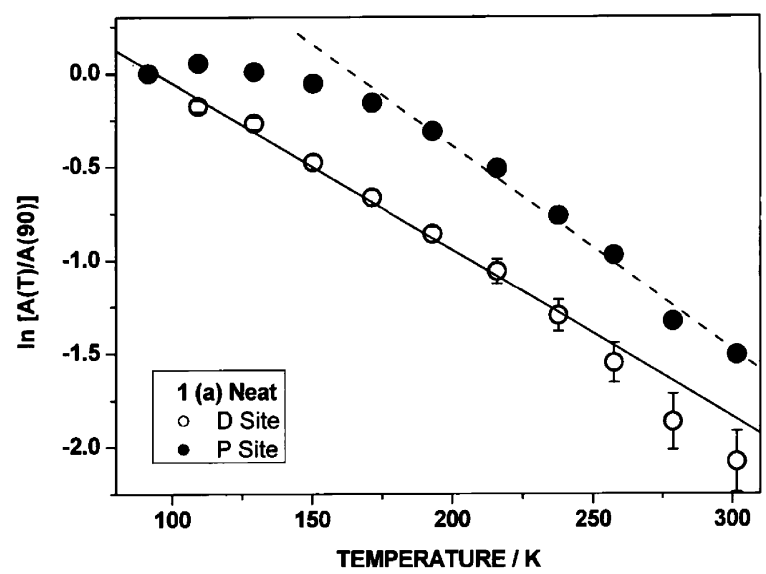

Fig. 6 Comparison of the temperature dependence of the recoil-free fraction for the two iron sites (diamagnetic, open data points and paramagnetic, filled data points) of $2(a)$.

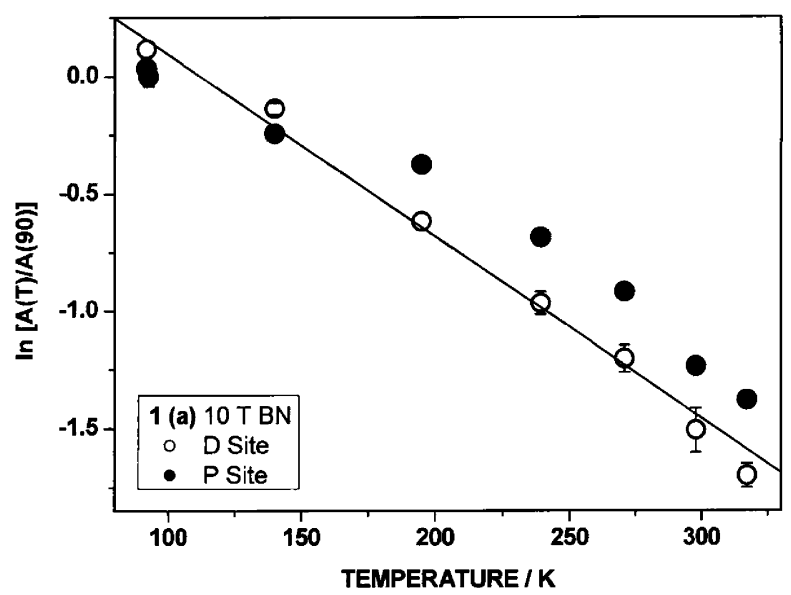

Fig. 7 Comparison of the temperature dependence of the recoil-free fraction of the two iron sites in a pressed pellet (10 tons, BN matrix) of $2(a)$.

\section{Summary and conclusions}

The one-electron oxidation product 2 of triferrocenylmethanol (1) has been synthesized as the $\mathrm{PF}_{6}{ }^{-}$complex, and its crystal structure, electrochemical behavior and Mössbauer parameters have been determined. The crystal structure at $100 \mathrm{~K}$, monoclinic, space group $\mathrm{Pc}$, was refined to $R_{1}=0.0434$ and shows two distinct $\mathrm{Fe}-\mathrm{C}$ bond distances associated with the two kinds of 
iron atoms in the structure. The electrochemical properties of the cation show two quasi-reversible waves, similar to those observed in the neutral starting compound. The temperature-dependent Mössbauer data show two distinct Fe resonances associated with the two diamagnetic and the single paramagnetic site. The latter shows a spin-lattice relaxation behavior obeying an $\sim 2.6$ power law in $T$. There is no evidence for electron delocalization among the three iron sites in the temperature range $5<T<300 \mathrm{~K}$.

\section{Notes and references}

1 A. Togni, and T. Hayashi, ed., Ferrocenes - Homogeneous Catalysis, Organic Synthesis, Materials Science, VCH, Weinheim, 1995.

2 M. Laskoski, W. Steffen, M. D. Smith and U. H. F. Bunz, Chem. Commun., 2001, 691-692.

3 P. Stepnicka, ed., Ferrocenes: Ligands, Materials and Biomolecules, John Wiley \& Sons, Chichester, 2008.

4 P. L. Pauson and W. E. Watts, J. Org. Chem., 1962, 27, 3880-3886.

5 A. N. Nesmeyanov, E. G. Perevalova, L. I. Leont'eva and Y. A. Ustynyuk, Isv. Akad. Nauk SSSR Ser. Khim., 1966, 558-559, (Bull. Acad. Sci. USSR Div. Chem. Sci. (Engl. Transl.), 1966, 528-529).

6 A. N. Nesmeyanov, E. G. Perevalova and L. I. Leont'eva, Izv. Akad. Nauk SSSR Ser. Khim., 1973, 142-144, (Bull. Acad. Sci. USSR, Div. Chem. Sci. (Engl. Transl.), 1973, 22, 141).

7 B. Wrackmeyer, U. Dörfler, W. Milius and M. Herberhold, Z. Naturforsch B, 1995, 50b, 201-204.

8 J. R. Garabatos Perera, R. Wartchow and H. Butenschön, J. Organomet. Chem., 2004, 689, 3541-3549.

9 J. R. Garabatos-Perera and H. Butenschön, J. Organomet. Chem., 2008, 693, 357-360.

10 J. R. Garabatos-Perera and H. Butenschön, J. Organomet. Chem., 2009, 694, 2047-2052.

11 J. R. Garabatos-Perera, R. Wartchow and H. Butenschön, Adv. Synth. Catal., 2009in press.
12 J. M. McBride, Tetrahedron, 1974, 30, 2009-2022.

13 H. Lankamp, W. T. Nauta and C. MacLean, Tetrahedron Lett., 1968, 9, 249-254.

14 S. Cohen, J. Ma, H. Butenschön and R. H. Herber, Dalton Trans., 2009, 6606-6609.

15 G. Werner, C. W. Lehmann and H. Butenschön, Adv. Synth. Catal., 2010, 352, 1345-1355.

16 G. M. Sheldrick, Acta Crystallogr., Sect. A: Found. Crystallogr., 2008, 64, 112-122.

17 R. H. Herber and I. Nowik, J. Nucl. Radiochem. Sci., 2008, 9, 33-36.

18 http://www.megadaq.com.

19 CCDC 804181 contains the supplementary crystallographic data for this paper. These data can be obtained free of charge from The Cambridge Crystallographic Data Centre via www.ccdc.cam.ac.uk/data_request/cif.

20 R. Martinez and A. Tiripicchio, Acta Crystallogr., Sect. C: Cryst. Struct. Commun., 1990, 46, 202-205.

21 K. Venkatasubbaiah, A. Doshi, I. Nowik, R. H. Herber, A. L. Rheingold and F. Jäkle, Chem.-Eur. J., 2008, 14, 444-458.

22 H. Schottenberger, K. Wurst, U. J. Griesser, R. K. R. Jetti, G. Laus, R. H. Herber and I. Nowik, J. Am. Chem. Soc., 2005, 127, 6795-6801.

23 A. Abragam, and B. Bleaney, Electron Paramagnetic Resonance of Transition Ions (International Series of Monographs on Physics), Clarendon Press, Oxford, 1970.

24 N. N. Greenwood, and T. C. Gibb, Mössbauer Spectroscopy, ChapmanHall, London, 1971.

25 R. H. Herber, ed., Chemical Mössbauer Spectroscopy, Plenum Press, New York, 1985.

26 R. H. Herber and I. Nowik, J. Organomet. Chem., 2008, 693, 30073010.

27 F. Parak and H. Formanek, Acta Crystallogr., Sect. A: Cryst. Phys., Diffr., Theor. Gen. Crystallogr., 1971, 27, 573-578.

28 F. Parak, H. Hartmann, K. D. Aumann, H. Reuscher, G. Rennekamp, H. Bartunik and W. Steigemann, Eur. Biophys. J., 1987, 15, 237-249.

29 V. S. Kumar, S. Wan, D. L. Aubele and P. E. Floreancig, Tetrahedron: Asymmetry, 2005, 16, 3570-3578.

30 D. Gelman, personal communication to R. H. H. 